\title{
The effect of evening primrose oil on fatigue and quality of life in patients with multiple sclerosis
}

This article was published in the following Dove Press journal:

Neuropsychiatric Disease and Treatment

\section{Nastaran Majdinasab, ' Foroogh Namjoyan, ${ }^{2}$ Mohsen Taghizadeh, ${ }^{3}$ Hamid Saki ${ }^{4}$ \\ 'Department of Neurology, Ahvaz Jundishapur University of Medical Sciences, Musculoskeletal Rehabilitation Research Center, Ahvaz, Iran; ${ }^{2}$ Department of Pharmacognosy, Marine Natural Pharmaceutical Research Center, School of Pharmacy, Ahvaz Jundishapur University of Medical Sciences, Ahvaz, Iran; ${ }^{3}$ Department of Nutrition, Research Center for Biochemistry \& Nutrition in Metabolic Diseases, Kashan University of Medical Sciences, Kashan, Iran; ${ }^{4}$ Department of Neurology, Ahvaz Jundishapur University of Medical Sciences, Ahvaz, Iran}

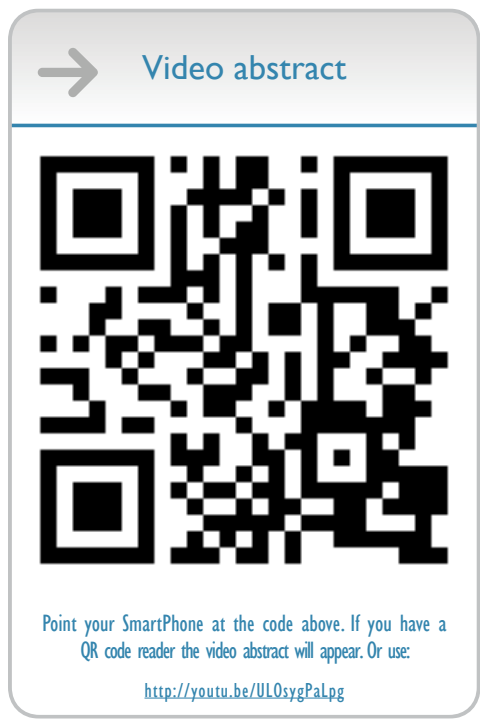

Correspondence: Hamid Saki Department of Neurology, Ahvaz Jundishapur University of Medical Sciences, Golestan Street, Golestan Hospital, Ahvaz, Iran

Tel +98916 6168500

Email hamidsaki5555@gmail.com
Background: Multiple sclerosis (MS) is a chronic progressive and inflammatory disease of the central nervous system that is characterized by demyelination in the central nervous system. In regard to the prevalence of diseases and enormous costs imposed on society and the health system, finding a way to stop the progression of the disease using drugs with fewer side effects seems a serious sanitation issue to the health of the international community. This study aimed to evaluate the effect of evening primrose oil (EPO) on fatigue and quality of life in patients with MS.

Materials and methods: In this double-blind randomized clinical trial, 52 patients with MS were chosen and categorized into 2 groups which received 2 doses of EPO and placebo. In addition, the quality of life and fatigue scale in these patients were investigated before the treatment and again 3 months after therapy. The findings were then compared between the 2 groups.

Result: EPO consumption significantly increased cognitive function, vitality, and overall life satisfaction and also reduced pain and fatigue compared to placebo $(P<0.05)$.

Conclusion: Our findings indicated that EPO consumption had no impact on the quality of life in general; however, it had a significant effect on several important aspects of life quality such as the increase of cognitive function, vitality, and overall life satisfaction. It also reduced the pain and fatigue in comparison to the placebo consumption. Herbal medicines are brittle and have fewer side effects than chemical drugs. With use of this plant, reduced fatigue and improved quality of life were observed in MS patients. But the drug did not prevent the progression of the disease.

Keywords: cognitive function, vitality, overall life satisfaction, pain, fatigue

\section{Introduction}

Multiple sclerosis (MS) is a chronic progressive inflammatory disease of the central nervous system (CNS) that is characterized by demyelination in the CNS and leads to inability of individuals (one of the most important pathological features of MS is demyelination. The pathology of axonal can be raised but in general pathology demyelination is more relevant. ${ }^{1,2}$ Two to three million people are affected by MS worldwide, and every week about 200 new people begin suffering from MS. Eighty percent of people with this disorder have some degree of disability. ${ }^{3}$ The prevalence of MS in various geographical areas is different. This disease is widespread in Europe, Canada, America, New Zealand, and South Australia and is less commonly seen in Asia. ${ }^{4-6}$ In recent years, studies in the Middle East and Iran indicate a high prevalence of the disease in these regions. ${ }^{3}$ In Iran, the prevalence of this disease has been reported 30-40 per 100,000 persons (Iran was previously only a low-prevalence area for MS, but recently, with increasing prevalence of Iran, it has been classified as an intermediate region). ${ }^{3}$ In most countries, economic concerns due to this disease are 
more significant than stroke and Alzheimer because MS is more common in young individuals and also because this is a chronic and long-term illness, per se. The annual costs in America have been estimated at \$5.2 million. ${ }^{3}$

The cause of the disease is still not fully recognized, and various theories are presented in this regard. Items such as heredity, environmental factors, and even viruses can be introduced as the underlying causes of the disease. ${ }^{7-9}$ There is much evidence that it is an autoimmune disease.

Studies have shown that in these patients inflammatory factor levels (CRP) are increased and these factors have myelotoxic property directly and cause demyelination and axonal damage. ${ }^{?}$

Among the environmental factors, nutritional factors are recognized as one of the most important of possible reasons in MS outbreak. Although available epidemiological studies have provided conflicting evidence, these investigations point to the role of some factors in control and prevention of MS disease such as vitamin D, polyunsaturated fatty acids, particularly omega-3 and omega-6, antioxidants such as vitamin $\mathrm{C}$, dietary fiber, vegetative protein, fish, juice, and whole grains; however, other factors such as saturated and animal fat, red meat, and high-fat dairy are the known risk factors in this disease..$^{4,10,11}$

In regard to the increasing rate of mortality, issues such as enormous costs, various problems of MS patients, taking care of these patients, and attempts to decrease the difficulties and symptoms of MS disease are all essential.

Today, medicinal plants are economically important and are used raw or processed in modern and traditional medicine. ${ }^{12}$ The use of herbal products is increasing worldwide, and the World Health Organization studies indicate that $80 \%$ of the world population is dependent on plants in terms of medical aspects. ${ }^{13}$

Evening primrose, with the scientific name of Oenothera biennis, belongs to the family of panacea plants. ${ }^{14}$ Evening primrose as a medicinal plant has a long history and has a high potential in terms of producing gamma-linolenic acid (GLA). Mature and dried seeds constitute medicinal aspect of this plant, containing the effective oil substance. ${ }^{15}$ This plant oil contains an essential fatty acid called GLA acid which is an important intermediate in the human body metabolism. Evening primrose oil (EPO) consists of 98\% triacylglycerol, and about $1 \%-2 \%$ of nonsoap material and some important sterols and tocopherols. The major fatty acids in EPO are palmitic acid (7\%-10\%), stearic acid (1/5\%-3/5\%), oleic acid $(6 \%-11 \%)$, linoleic acid $(65 \%-80 \%)$, and $\gamma$-linolenic acid (8\%-14\%). ${ }^{16,17}$ The findings of previous studies revealed that linoleic acid and $\gamma$-linolenic acid are able to prevent prostaglandins synthesis. ${ }^{14}$

The main origin of this plant is South America and Central America. The brewed root of this plant is used in these regions against obesity and intestinal pain. Evening primrose seed oil is very effective in reducing menstrual pain and in the treatment of diabetes mellitus and for reducing cholesterol. Useful therapeutic effects of this plant are also significantly observed with regard to the symptoms of irritable bowel, and for blood circulation disorders and rheumatism. ${ }^{18}$

In regard to the prevalence of the disease and enormous costs imposed on society and the health system, finding a way to stop the progression of the disease using drugs with fewer side effects seems a serious issue in the health of international community. Therefore, this study aims to evaluate the effect of EPO on fatigue and quality of life in patients suffering from MS. Our goal was to prevent the progression of symptoms and to improve some of the symptoms, but preventing the transformation of relapsing/remitting MS to progressive was not a goal of the study.

The purpose of this study was to evaluate the effect of EPO on fatigue and quality of life, but a comparison of the effect of this oil on these 2 factors (fatigue and quality of life) was not a goal of the study.

The following subscales were used:

1. Physical health: This scale evaluates the individual in terms of physical condition and was evaluated using 12 questions in the Multiple Sclerosis Quality of Life (MSQL-54) questionnaire.

2. Role playing in relation to physical status: This scale evaluates the limitations that follow for a person's physical condition, assessed by 4 questions in the questionnaire.

3. Role in relation to psychological state: This scale evaluates the limitations that follow a person's mental status, assessed by 4 questions in the questionnaire.

4. Pain: This scale evaluates the generalized pain caused by MS in patients, which was evaluated by 3 questions in the questionnaire.

5. Vitality: This scale evaluates happiness the person's feels and was evaluated by 4 questions in the questionnaire.

6. Energy: This scale assesses the individual's energy and energy for doing routines and extra tasks, which was evaluated by 6 questions in the questionnaire.

7. Understanding general health: This scale assesses whether a person understands his own health and whether a person knows what health is, and was evaluated by 4 questions in the questionnaire. 
8. Social function: This scale assesses the social constraints imposed upon by the disease on the individual, and was evaluated by 6 questions in the questionnaire.

9. Cognitive function: This scale assesses how the disease MS affects the memory and concentration of the person. It was assessed by 4 questions in the questionnaire.

10. Health threat: This scale examines how much the disease can threaten a person's health. It was assessed by 4 questions in the questionnaire.

11. Sexual function: This scale measures the ability of a person's sexual function to determine how much the disease can affect sexual function of the individual, which was evaluated by 5 questions in the questionnaire.

12. Total individual satisfaction of life: This scale determines how much this disease can affect the overall satisfaction of one's life. It was assessed by 2 questions in the questionnaire.

\section{Literature review}

New research showed that non-triglyceride fraction of unrefined EPO to contain triple-oxidated trans-caffeoy derivatives of betulinic, morolic, and oleanolic acid. These lipophilic pentacyclic triterpenes have free radical scavenging, cyclooxygenase and neutrophil elastase inhibitory activities. Thus, this may be useful for human health. Nevertheless, laboratory analysis has shown that these lipophilic antioxidants are seen only in trace quantities in EPO commercial samples. Due to useful effect of triterpene esters, scientists postulate that this product is likely to have better health benefits than

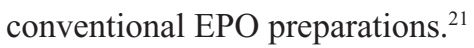

The result of new studies demonstrated that myalgic encephalomyelitis has a relationship with viral infection. Also, it must be pointed out that such infections, by inhibiting the delta- 6 desaturation (D6D) of the precursor essential fatty acids including linoleic acid and $\alpha$-linolenic acid, impair the ability of the person to biosynthesize long-chain polyunsaturated fatty acids, thereby leading to an adverse effect on the biosynthesis of eicosanoids from the chain polyunsaturated fatty acids dihomo- $\gamma$-linolenic acid, arachidonic acid, and eicosapentaenoic acid, which fits in well with the symptoms and signs of myalgic envephalomyelitis. So, virgin coldpressed non-raffinated EPO was suggested for treatment in patients with the disease. ${ }^{22}$

In one study, 90 patients with Sjogren's syndrome entered a 6-month study for treatment with EPO. By assessing using the Visual Analog Scale, it was found that no statistically significant improvement was observed in fatigue during a 24 -hour period between the 2 groups. Finally, the authors suggested that EPO for fatigue in primary Sjogren's syndrome is ineffective. ${ }^{23}$

In post-viral fatigue syndrome patients, the researchers studied the effect of essential fatty acid therapy. The preparation that they used contained linoleic acid, eicosapentaenoic acid, and docosahexaenoic acid, and the patients received either the preparation or a placebo. There were no adverse events reported. They suggested that essential fatty acids provide an effective, rational, and safe treatment for this syndrome. $^{24}$

EPO is taken orally in the form of a gel capsule and has been used for breast pain and atopic dermatitis treatment. ${ }^{27,28}$ EPO is necessary for many functions, but there is not enough evidence to prescribe its use for the relief of atopic dermatitis or breast pain. ${ }^{25}$

\section{Materials and methods}

Due to the high prevalence of fatigue in MS patients, most of our patients were also tired of the Modified Fatigue Impact Scale (MFIS). Apart from depression, other secondary causes of fatigue included endocrine abnormalities; liver, heart, and pulmonary conditions, and autoimmune diseases, and people whose fatigue caused them the abovementioned symptoms were excluded.

Before the implementation of the plan, capsules containing $1 \mathrm{~g}$ of EPO as well as placebo capsules were provided in a predetermined number.

Method of preparation of the capsule: The soft gelatin capsule of the evening primrose and placebo was placed on the market by Barije Pharmaceutical Company, Kashan, Iran.

Gloricum oil was standardized on the basis of its GLA content using standard chromatography gas apparatus, (GC Clarus 680 Chromatography Machine, made by Perkin Elmer, Waltham, MA, USA). The characteristics of the column were as follows:

1. Fixed phase Wcot Fused Silica Type CP-Column 7753 (CP-WAX 52 CB).

2. Dimensions of the pillar: column length: $50 \mathrm{~m}$, inner diameter: $0.32 \mathrm{~mm}$, outer diameter: $0.45 \mathrm{~mm}$, and film thickness: $0.20 \mu \mathrm{m}$.

3. For chromatography, the moving phase had a constant speed of $1 \mathrm{~mL} / \mathrm{min}$, injector output was $250^{\circ} \mathrm{C}$, the temperature of the detector was $250^{\circ} \mathrm{C}$, injection rate was $1 \mu \mathrm{L}$, spilite was 50 , and the temperature program used was as follows: starting at $170^{\circ} \mathrm{C}$ with increase of $3^{\circ} \mathrm{C} / \mathrm{min}, 20$ minutes at $230^{\circ} \mathrm{C}$, for total time 40 minutes. 
Then soft gelatin capsule with gelatin shell coating was made by softgel coating. After drying, it was poured into the flacon, and following quality control checks was given to the project executives.

In this double-blind randomized clinical trial (both the patient and the donor are unaware of the type of medication [placebo or EPO], and the sampling method used was a randomized one with 4-way permutation sequences [a statistical method for sampling]), 52 MS patients whose disease was approved through standard diagnostic procedures were studied. These patients were chosen among the people who were admitted to Golestan Hospital, a part of the MS society of Ahwaz.

Diagnosis of the disease was based on neurological examinations and MRI by a neurologist.

Included patients were referred from a neurology specialist's office and should have fulfilled the following criteria:

1. Diagnosis of the disease based on neurological examinations and MRI by a neurologist.

2. $18-55$ years old.

3. Expanded Disability Status Scale (EDSS) score $<6$.

4. No other neurological disease, such as stroke or psychiatric condition, such as depression.

5. Women who were not pregnant.

6. Absence of diseases such as cancer, autoimmune diseases such as lupus or rheumatoid arthritis, and heart disease.

7. Not having suffered a new attack in the past month and had not use pulse corticosteroids.

The parameters that were influenced by the EPO are really different, which is significant.

Ahwaz Jundishapur University's ethics committee approved this research, and all patients were deliberately enrolled. Before entering the study, written informed consent was obtained from all patients.
The biographical information of these patients was recorded, and they were asked to complete a questionnaire regarding life quality of Iranians with MS disease. The questionnaires used were Multiple Sclerosis Quality Of Life (MSQOL)-54 and MFIS. The use of multiple different pain scales was avoided. Based on the MFIS questionnaire, we compared fatigue into two groups (experimental and control) but did not compare the fatigue of components separately (physical, cognitive and psychosocial) in the two groups.

Patients were randomly divided into 2 groups.

The experimental group: Patients were asked to take $1 \mathrm{~g}$ oral capsule containing EPO every 12 hours for 3 months in addition to the standard treatment for their disease.

Control group: Patients were administered the standard medicinal therapy for disease along with placebo.

After 3 months, all the patients were asked to return to the medical center and retake the MSQOL-54 and MFIS questionnaires.

The results of these questionnaires were statistically analyzed.

Table 1 shows the patient's clinical and demographic characteristics.

\section{Statistical analysis}

After the results were obtained through tests and questionnaires, the required data were statistically analyzed using SPSS, version 22 (IBM Corporation, Armonk, NY, USA). Descriptive statistics were also utilized in order to analyze the dataset.

Covariance analysis was then employed, since in addition to the independent variable (2 groups of drug and placebo) and the dependent variable (quality of life and fatigue in

Table I Patient's clinical and demographic characteristics

\begin{tabular}{|c|c|c|c|c|}
\hline Study group & \multicolumn{2}{|c|}{ Control group } & \multicolumn{2}{|c|}{ Experimental group } \\
\hline Number & \multicolumn{2}{|c|}{26 patients } & \multicolumn{2}{|c|}{26 patients } \\
\hline \multirow[t]{2}{*}{ Gender } & Female & Male & Female & Male \\
\hline & 18 & 8 & 20 & 6 \\
\hline Age & \multicolumn{2}{|c|}{ 20-55 years old } & \multicolumn{2}{|c|}{$20-55$ years old } \\
\hline Disease duration & \multicolumn{2}{|c|}{$1-5$ years } & \multicolumn{2}{|c|}{$1-5$ years } \\
\hline MS type & \multicolumn{2}{|l|}{ RRMS } & \multicolumn{2}{|l|}{ RRMS } \\
\hline Disease modifying treatment & \multicolumn{2}{|c|}{ Interferon $\beta$ Ia (cinovex) or glatiramer } & \multicolumn{2}{|c|}{ Interferon $\beta$ la (cinovex) or glatiramer } \\
\hline EDSS & \multicolumn{2}{|c|}{$<6$ and more patients were $\mathrm{I}-3$} & \multicolumn{2}{|c|}{$<6$ and more patients were $\mathrm{I}-3$} \\
\hline Depression score & \multicolumn{2}{|c|}{ 0-7 normal } & \multicolumn{2}{|c|}{$0-7$ normal } \\
\hline
\end{tabular}

Note: Depression scores in patients ranging from 0 to 7 were considered in the normal range and were included in the study, but those with a depression score over 7 were considered depressed and were not included in the study from the beginning.

Abbreviations: RRMS, relapsing/remitting multiple sclerosis; EDSS, Expanded Disability Status Scale; MS, multiple sclerosis. 
patients 3 months after treatment), there was a third variable called covariate (pretest) (fatigue and quality of life in patients before treatment) in this study.

The basic assumptions for all covariance tests were the normality, homogeneity of variance, and homogeneity of regression slope.

Kolmogorov-Smirnov test was used for checking the normality, and Levene's test was employed in order to evaluate the homogeneity of variance.

The significant level was $P<0.05$.

The results obtained from the assumptions of normality and homogeneity of variances indicated no significant deviation. In order to examine the differences between quality of life and fatigue variables between the experimental and control groups, analysis of covariance was employed.

The dependent variables were the quality of life and fatigue in patients 3 months after treatment, and control variables or covariates were the quality of life and fatigue in patients before treatment.

The basic assumptions, normality, and homogeneity of variances, were evaluated. No significant interaction between covariate and independent variables was seen, which reveals that there was no interaction between these 2 variables. Therefore, the assumption of homogeneity regression line slopes was met.

\section{Results}

The findings obtained from the covariance analysis showed that there was a significant difference between the average of vitality scale, pain scale, overall satisfaction with life, cognitive function, and fatigue in the experimental group compared with the control group 3 months after the initiation of treatment.

According to the results, at the significance level of 0.05 , there was a significant difference between the average of vitality scale after 3 months of treatment in both groups, control (placebo) and experimental, $P=0.024$. This meant that the independent variable (group) had a significant effect (Figure 1).

With reference to the results at the significant level of 0.05 , there was a significant difference between the average results seen on the pain scale after 3 months of treatment in both groups, control (placebo) and experimental, $P<0.001$. In other words, the effect of the independent variable (group) was significant (Figure 2).

According to the results at the significant level of 0.05 , there was a significant difference between the average scores on the cognitive functioning scale after 3 months of

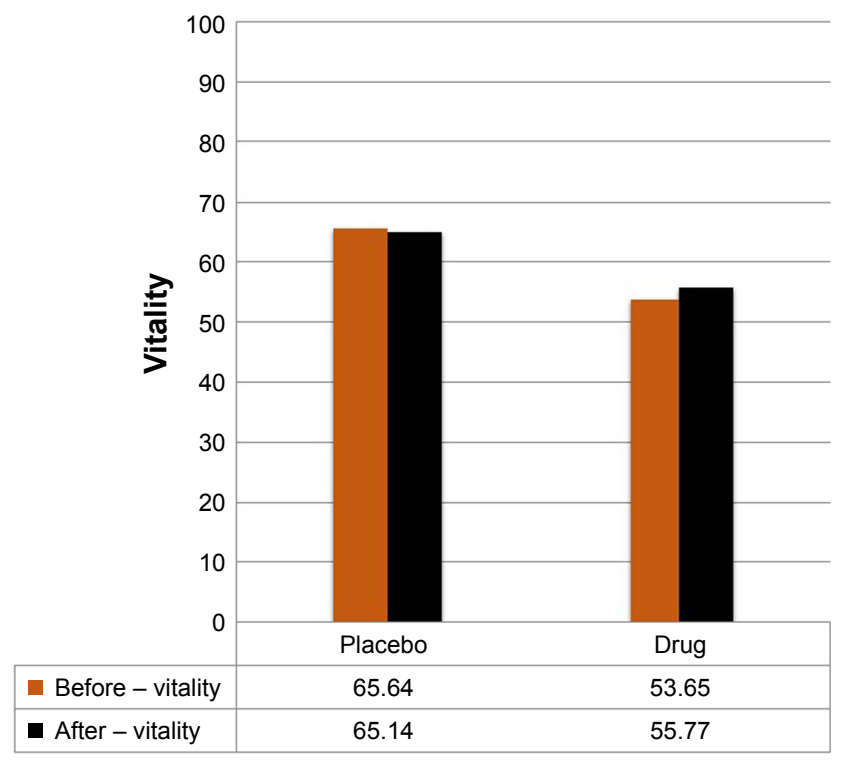

Figure I Comparison of vitality before treatment and 3 months after treatment in the 2 groups, control (placebo) and experimental.

treatment in both control (placebo) and experimental groups, $P<0.001$. In other words, the independent variable (group) had a significant effect (Figure 3 ).

With reference to the results at the significant level of 0.05 , there was a significant difference between the average score of overall satisfaction with life after 3 months of treatment in both control (placebo) and experimental groups, $P<0.001$. In other words, the effect of the independent variable (group) was significant (Figure 4).

According to the results at the significant level of 0.05 , there was a significant difference between the average scores

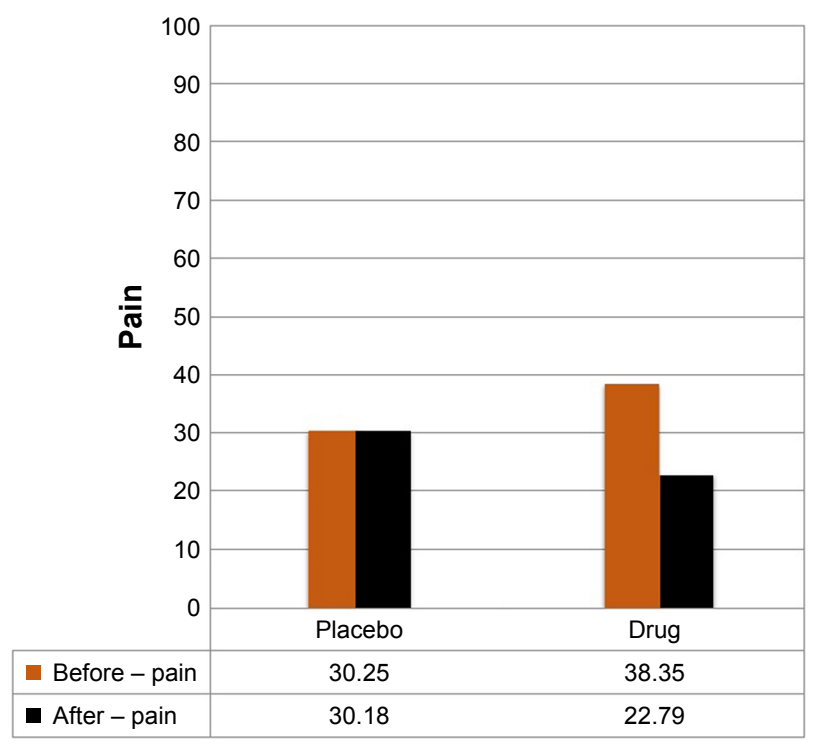

Figure 2 Comparison of scores on the pain scale before treatment and 3 months after treatment in the 2 groups, control (placebo) and experimental. 


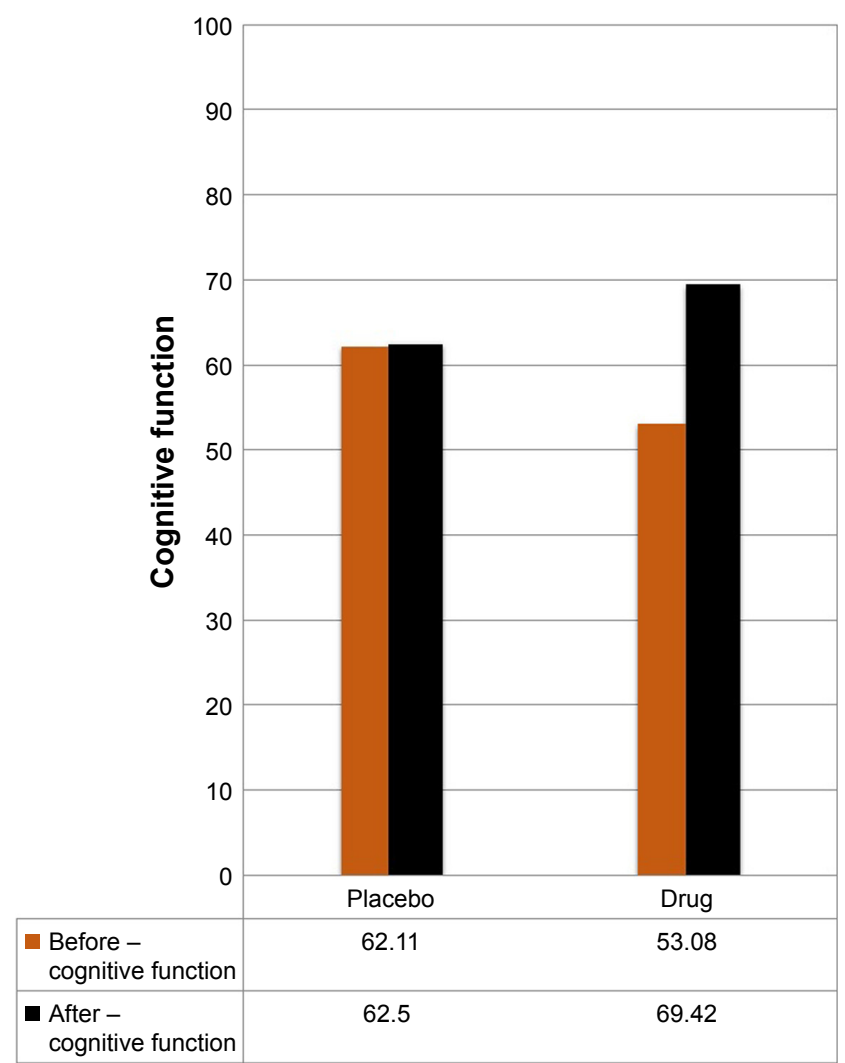

Figure 3 Comparison of cognitive function before treatment and 3 months after treatment in both groups, control (placebo) and experimental.

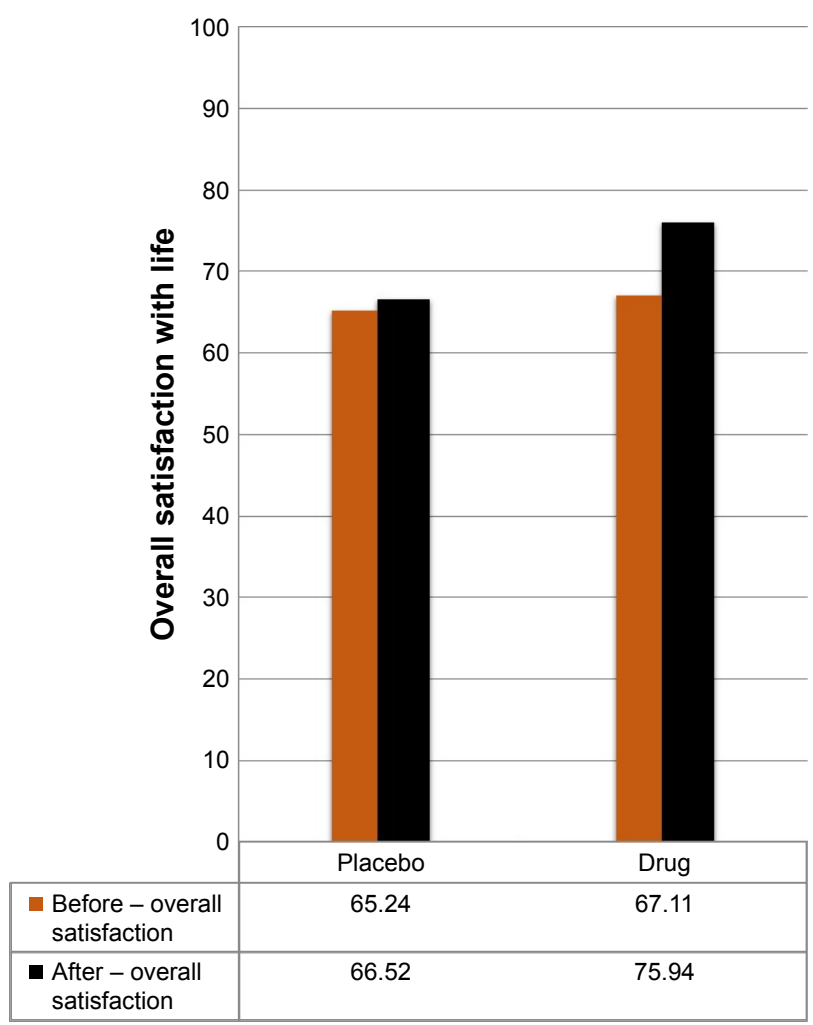

Figure 4 Comparison of overall life satisfaction before treatment and 3 months after treatment in both groups, control (placebo) and experimental.

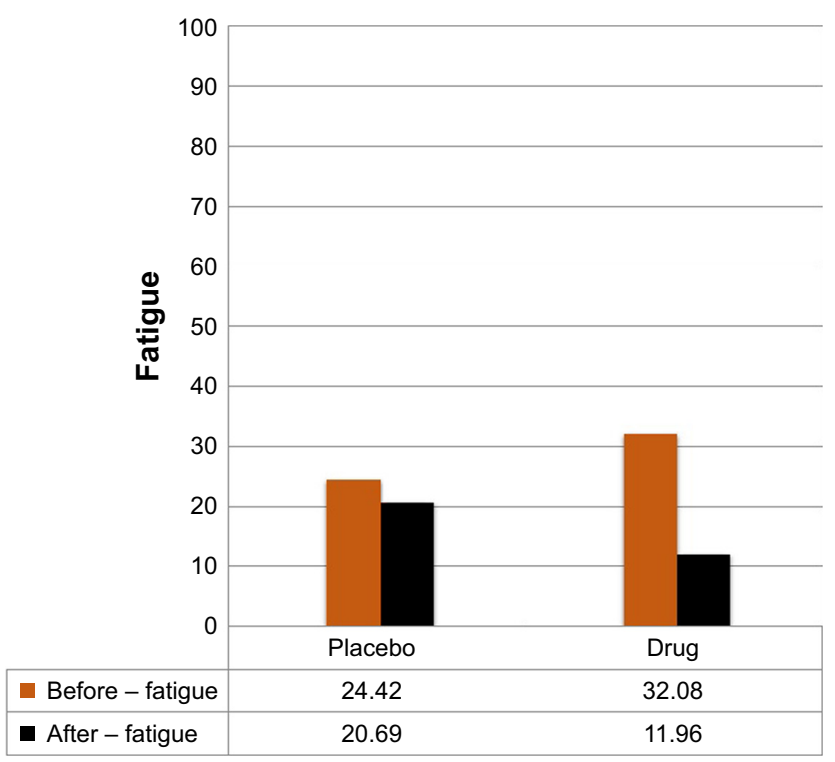

Figure 5 Comparison of fatigue scale scores before treatment and 3 months after treatment in both groups, control (placebo) and experimental.

on the fatigue scale after 3 months of treatment in both control (placebo) and experimental groups, $P<0.001$. In other words, the independent variable (group) had a significant effect (Figure 5).

However, there was no significant difference between the average scores of physical health, role in relation to health, playing a role in mental status, social functioning, energy, sexual function, health perception, and health hazards in the experimental group compared with the control group 3 months after the treatment $(P>0.05)$.

The results also indicated there was a significant effect of the control (covariate) variable $(P<0.05)$.

\section{Overall results}

It can be claimed that after removing the effect of the control variable (covariate), a significant different was found between the average scores of vitality, pain, overall life satisfaction, cognitive function, and fatigue after 3 months of treatment in both control (placebo) and experimental groups (EPO consumers).

Therefore, the use of EPO after 3 months in the experimental group led to an increase in vitality, overall satisfaction with life, and cognitive function and a reduction in scores on the pain scale and fatigue scale significantly.

The comparison of pain scale, vitality, overall life satisfaction, cognitive function, and fatigue before treatment and 3 months after treatment in the 2 groups, control and experimental, is illustrated in Figures 1-5. 
The study we performed was unique, and there is no similar study available for comparison of the results with, and there were only 5 relatively similar studies that we found upon literature review.

\section{Discussion}

The cause of MS is still not fully recognized, and various theories are presented in this regard. There is much evidence that it is an autoimmune disease.

The findings obtained through this study revealed that the EPO consumption in MS disease had no impact on quality of life in general; however, there was a significant effect on 4 important aspects of quality of life in comparison to the consumption to patients who consumed the placebo: increase of cognitive function, vitality, overall satisfaction with life, and decrease in pain. Moreover, the use of EPO in comparison with placebo consumption had a significant effect on reduction of fatigue scale scores $(P<0.05)$.

Rezapour-Firouzi et a ${ }^{19}$ examined the efficacy of a combination drug containing hemp and evening primrose in order to reduce EDSS and the level of liver enzymes in 23 patients with MS and showed that this drug has beneficial effects in improving the clinical symptoms.

Parameters influenced by EPO are really different, which is noteworthy. In our study, we declared that the parameters of pain, vitality, fatigue, cognitive impairment, and quality of life in general were modified by EPO. It has been shown that Rezapour-Firouzi et $\mathrm{al}^{20}$ worked on EPO and cannabis and concluded that $\mathrm{EPO}$ and cannabis have beneficial effects on the improvement of EDSS score and the activity of liver enzymes in MS patients. Alvandipour et $\mathrm{al}^{26}$ studied EPO and Vitamin E, and they came to the conclusion that vitamin $\mathrm{E}$ and $\mathrm{EPO}$ have similar therapeutic effects in treating periodic breast pains and that it can be used as therapeutic alternatives in this area.

\section{Conclusion}

To the best of our knowledge, this study is unique in terms of the examination of the effect of EPO on life quality and fatigue in patients with MS and the novel use of questionnaire. During the study, we stated several times that we used questionnaires to investigate the effect of EPO on fatigue and quality of life of MS patients. We could not use other scales because of the controversy with the title of the paper. The values in the placebo group were identical before and after treatment and we did not have any interactions in the numbers, and indeed the values were close or identical.

Considering the findings obtained in this study that EPO has a significant effect on 4 aspects of quality of life and fatigue in comparison to the placebo, using a larger drug dosage and increasing the duration of the study (more than 3 months) are recommended for future studies. Besides, the lack of longterm follow-up of patients was the limitation of this study.

In addition to the above limitation, our study also did not take into consideration MS patients younger than 18 years of age.

Approximately $80 \%-97 \%$ of patients with MS complain of fatigue, and while the real cause of disease is unknown, nonpharmacological treatments for reducing fatigue include taking cold drinks, aerobic exercise, and resting. Many clinical studies examine the effect of fatigue control with the use of CNS modifiers such as methylphenidate, modafinil, and pumilein, and results have been proven. In addition, factors such as buprupion, sertraline, amantadene, and aminopyridine have also been shown to reduce fatigue due to MS.

\section{Author contribution}

All authors contributed toward data analysis, drafting and critically revising the paper and agree to be accountable for all aspects of the work.

\section{Disclosure}

The authors report no conflicts of interest in this work.

\section{References}

1. Frohman EM, Racke MK, Raine CS. Multiple sclerosis the plaque and its pathogenesis. New Eng J Med. 2006;354(9):942-955.

2. Pugliatti M, Rosati G, Carton H, et al. The epidemiology of multiple sclerosis in Europe. Eur J Neurol. 2006;13(7):700-722.

3. Ghafari S, Ahmadi F, Nabavi M. [Effect of calming technique on fatigue of muscle in multiple sclerosis patients]. Med J Shahrekord Univ. 2009;100:61-68. Persian.

4. Ascherio A, Munger K. Epidemiology of multiple sclerosis: from risk factors to prevention. Semin Neurol. 2008;28(1):17-28.

5. Etemadifar M, Janghorbani M, Shaygannejad V, Ashtari F. Prevalence of multiple sclerosis in Isfahan, Iran. Neuroepidemiology. 2006; 27(1):39-44

6. Kantarci O, Wingerchuk D. Epidemiology and natural history of multiple sclerosis: new insights. Curr Opin Neurol. 2006;19(3):248-254.

7. Chataway J, Feakes R, Coraddu F, et al. The genetics of multiple sclerosis: principles, background and updated results of the United Kingdom systematic genome screen. Brain. 1998;121(Pt 10):1869-1887.

8. Robertson NP, Fraser M, Deans J, Clayton D, Walker N, Compston DA. Age adjusted recurrence risks for relatives of patients with multiple sclerosis. Brain. 1996;119(Pt 2):449-455.

9. Weinshenker BG, Bass B, Rice GPA, et al. The natural history of multiple sclerosis: a geographically based study I. Clinical course and disability. Brain. 1989;112(Pt 1):133-146.

10. Antonovsky A, Leibowitz URI, Smith HA, et al. Epidemiologic study of multiple sclerosis in Israel: I. An overall review of methods and findings. Arch Neurol. 1965;13(2):183-193.

11. Malosse D, Perron H, Sasco A, Seigneurin JM. Correlation between milk and dairy product consumption and multiple sclerosis prevalence: a worldwide study. Neuroepidemiology. 1992;11(4-6):304-312. 
12. Razzaque MA, Talukder NM, Islam MS, Bhadra AK, Dutta RK. The effect of salinity on morphological characteristics of seven rice (Oryza sativa) genotypes differing in salt tolerance. Pak J Biolog Sci. 2009;12(5):406-412.

13. Zargari A. Medicinal Plants. Vol 2, 5th ed. Tehran: Tehran University Press; 1991. Persian.

14. Bekhradi R. Giah Darmani Novin. Tehran: Motarjem Publication; 2004. Persian.

15. Ghasemnezhad A. Investigations on the Effects of Harvest Methods and Storage Conditions on Yield, Quality and Germination of Evening Primrose (Oenothera biennis L.) Seeds [dissertation]. Giessen, Germany: Justus Liebig University Giessen; 2007.

16. Christie WW. The analysis of evening primrose oil. Ind Crops Prod. 1999;10(2):73-83.

17. Hudson BJF. Evening primrose (Oenothera spp.) oil and seed. J Am Oil Chem Soc. 1984;61(3):540-543.

18. Kiss A, Derwiska MG, Granica S. Quantitative analysis of biologically active polyphenols in evening primrose (Oenothera paradoxa) seeds aqueous extracts. Polish J Food Nutr Sci. 2011;61(2):109-113.

19. Rezapour-Firouzi S, Arefhosseini SR, Ebrahimi-Mamaghani M, et al. Activity of liver enzymes in multiple sclerosis patients with Hot-nature diet and co-supplemented hemp seed, evening primrose oils intervention. Complement Ther Med. 2014;22(6):986-993.

20. Rezapour-Firouzi S, Arefhosseini SR, Farhoudi M, et al. Association of expanded disability status scale and cytokines after intervention with co-supplemented hemp seed, evening primrose oils and hot-natured diet in multiple sclerosis patients. Bioimpacts. 2013;3(1):43.
21. Puri BK. The clinical advantages of cold-pressed non-raffinated evening primrose oil over refined preparations. Med Hypotheses. 2004;62(1): 116-118.

22. Puri BK. Long-chain polyunsaturated fatty acids and the pathophysiology of myalgic encephalomyelitis (chronic fatigue syndrome). J Clin Pathol. 2007;60(2):122-124.

23. Theander E, Horrobin DF, Jacobsson LT, Manthorpe R. Gammalinolenic acid treatment of fatigue associated with primary Sjögren's syndrome. Scand J Rheumatol. 2002;31(2):72-79.

24. Behan PO, Behan WM, Horrobin D. Effect of high doses of essential fatty acids on the postviral fatigue syndrome. Acta Neurol Scand. 1990;82(3):209-216.

25. Stonemetz D. A review of the clinical efficacy of evening primrose. Holist Nurs Pract. 2008;22(3):171-174.

26. Alvandipour M, Tayybi P, Alizadeh R, Khodabakhshi H. Comparison of evening primrose with vitamin $\mathrm{E}$ in the treatment of periodic mastalgia. J Babol Univ Med Sci. 2010;13(59):7-11.

27. Kalati M, Kashanian M, Jahdi F, Naseri M, Haghani H, Sheikhansari N. Evening primrose oil and labour, is it effective? A randomised clinical trial. J Obstet Gynaecol. 2018;9:1-5.

28. Bayles B, Usatine R. Evening primrose oil. Am Fam Physician. 2009;80:1405-1408.
Neuropsychiatric Disease and Treatment

\section{Publish your work in this journal}

Neuropsychiatric Disease and Treatment is an international, peerreviewed journal of clinical therapeutics and pharmacology focusing on concise rapid reporting of clinical or pre-clinical studies on a range of neuropsychiatric and neurological disorders. This journal is indexed on PubMed Central, the 'PsycINFO' database and CAS,

\section{Dovepress}

and is the official journal of The International Neuropsychiatric Association (INA). The manuscript management system is completely online and includes a very quick and fair peer-review system, which is all easy to use. Visit http://www.dovepress.com/testimonials.php to read real quotes from published authors. 